\title{
Profilin-1 contributes to cardiac injury induced by advanced glycation end-products in rats
}

\author{
DAFENG YANG ${ }^{1 *}$, WEIWEI LIU ${ }^{2 *}$, LIPING MA ${ }^{3}$, YA WANG $^{1}$, JING MA $^{1}$, MINNA JIANG $^{1}$, \\ XU DENG ${ }^{4}$, FANG HUANG $^{2}$, TIANLUN YANG ${ }^{1}$ and MEIFANG CHEN ${ }^{5}$ \\ ${ }^{1}$ Department of Cardiology, Xiangya Hospital, Central South University, Changsha, Hunan 410008; ${ }^{2}$ Department of \\ Cardiology, The First Hospital of Changsha, Changsha, Hunan 410005; ${ }^{3}$ Department of Cardiology, \\ Shandong Provincial Qianfoshan Hospital, Shandong University, Jinan, Shandong 250014; ${ }^{4}$ Department of \\ Cardiology, The Third Xiangya Hospital, Central South University, Changsha, Hunan 410006; ${ }^{5}$ Department of \\ Geriatric Medicine, Xiangya Hospital, Central South University, Changsha, Hunan 410008, P.R. China
}

Received September 24, 2016; Accepted July 20, 2017

DOI: $10.3892 / \mathrm{mmr} .2017 .7446$

\begin{abstract}
Cardiac injury, including hypertrophy and fibrosis, induced by advanced glycation end products (AGEs) has an important function in the onset and development of diabetic cardiomyopathy. Profilin-1, a ubiquitously expressed and multifunctional actin-binding protein, has been reported to be an important mediator in cardiac hypertrophy and fibrosis. However, whether profilin-1 is involved in AGE-induced cardiac hypertrophy and fibrosis remains to be determined. Therefore, the present study aimed to investigate the function of profilin-1 in cardiac injury induced by AGEs. The model of cardiac injury was established by chronic tail vein injection of AGEs (50 mg/kg/day for 8 weeks) in Sprague-Dawley rats. Rats were randomly assigned to control, AGEs, AGEs + profilin-1 shRNA adenovirus vectors (AGEs $+\mathrm{S}$ ) or AGEs + control adenovirus vectors (AGEs $+\mathrm{V}$ ) groups. Profilin-1 shRNA adenovirus vectors were injected via the tail vein to knockdown profilin-1 expression at a dose of $3 \times 10^{9}$ plaque forming units every 4 weeks. Echocardiography was performed to measure cardiac contractile function. Cardiac tissues were stained with Masson's trichrome stain to evaluate ventricular remodeling. The serum levels of procollagen type III N-terminal peptide were detected by ELISA. The expression of profilin-1, receptor
\end{abstract}

Correspondence to: Dr Tianlun Yang, Department of Cardiology, Xiangya Hospital, Central South University, 87 Xiangya Road, Changsha, Hunan 410008, P.R. China

E-mail: tianluny@163.com

Dr Meifang Chen, Department of Geriatric Medicine, Xiangya Hospital, Central South University, 87 Xiangya Road, Changsha, Hunan 410008, P.R. China

E-mail:22623007@qq.com

*Contributed equally

Key words: profilin-1, advanced glycation end-products, cardiac hypertrophy, cardiac fibrosis for AGEs (RAGE), Rho, p65, atrial natriuretic peptide, $\beta$-myosin heavy chain, matrix metalloproteinase (MMP)-2 and MMP-9 were determined using reverse transcription-quantitative polymerase chain reaction (RT-qPCR) and/or western blot analysis and immunohistochemistry staining. The results demonstrated that chronic injection of exogenous AGEs led to cardiac dysfunction, hypertrophy and fibrosis, as determined by echocardiography, Masson trichrome staining and the expression of associated genes. The expression of profilin-1 was markedly increased in heart tissue at the mRNA and protein level following AGE administration, as determined by RT-qPCR and western blotting, which was further confirmed by immunohistochemistry staining. Furthermore, the expression of RAGE, Rho and p65 was also increased at the protein level. Notably, knockdown of profilin-1 expression ameliorated AGE-induced cardiac injury and reduced the expression of RAGE, Rho and p65. These results indicate an important role for profilin-1 in AGE-induced cardiac injury, which may provide a novel therapeutic target for patients with diabetic heart failure.

\section{Introduction}

Diabetic cardiomyopathy (DCM), characterized by the presence of functional and structural abnormalities and not coronary heart disease, hypertension or other comorbidities, is the major cause of disability and mortality in patients with diabetes (1). This indicates that DCM may be a result of direct myocardial insult, distinguishing it from structural heart disease due to vascular complications. Identifying the mechanisms involved in DCM onset and progression is important for the development of therapeutic strategies to protect against diabetic heart failure. However, the pathogenesis of DCM remains to be fully elucidated. Increasing evidence has demonstrated that advanced glycation end products (AGEs), which are generated at an increased rate under chronic long-term hyperglycemia conditions, are a major contributor in the development and progression of DCM (2-8). Clinical studies have reported that, in diabetic tissues, AGEs accumulate to levels that are 14-fold higher compared with normal 
tissues, while serum levels of AGEs are associated with the degree of left ventricular function and myocardial blood flow reserve (2-4). AGEs increase the production of intracellular reactive oxygen species (ROS) via the receptor for AGEs (RAGE), which subsequently activates associated signaling cascades that promote cardiomyocyte hypertrophy (5), apoptosis (6) and myocardial fibrosis, ultimately resulting in diastolic and systolic dysfunction $(7,8)$. However, the underlying mechanisms responsible for myocardial injury triggered by AGEs remain to be fully elucidated.

It is established that profilin-1 is an evolutionarily conserved actin-binding protein that has an important function in the regulation of cytoskeleton dynamics by promoting actin polymerization (9). Profilin-1 also binds to polyphosphoinositide-based lipids and various proteins with poly-L-proline motifs, and serves an important role in the regulation of membrane trafficking, the small-GTPase signaling pathway, receptor activity and potentially transcriptional activity, which indicates that profilin-1 may be a central hub that controls molecular interactions $(10,11)$. An increasing amount of evidence has demonstrated that upregulated expression of profilin-1 is associated with endothelial dysfunction, vascular inflammation and remodeling under pathological stimulations, including AGEs (12), oxidized low-density lipoprotein (13) and angiotensin II $(14,15)$, and is not associated with high glucose (13). Furthermore, profilin-1 may also be secreted into the extracellular space, where it functions as an extracellular ligand leading to atherogenic effects and activates various signaling pathways, including the phosphatidylinositol 3-kinase/Akt and extracellular signal-regulated kinase $1 / 2$ pathways (16). In addition, it has been reported that profilin-1 is highly expressed in heart (17), and overexpression of profilin-1 promoted cardiac hypertrophy and contractile dysfunction in profilin-1 transgenic mice $(18,19)$, while knockdown of profilin-1 expression in spontaneous hypertensive rats attenuated cardiac hypertrophy and fibrosis (18). A number of additional studies have indicated that the abnormality of the cytoskeleton is an ultra-early change during left ventricular hypertrophy and fibrosis, and subsequent heart failure $(20,21)$. However, whether profilin-1 contributes to AGE-induced cardiac injury, and the underlying mechanism, remains unclear.

Due to the critical role of profilin-1 in actin dynamics, cytoskeletal reorganization and pathological vascular and cardiac hypotrophy, we hypothesize that profilin-1 may be involved in cardiac injury induced by AGEs, and that attenuated expression of profilin-1 may ameliorate AGE-induced adverse effects in the heart.

\section{Materials and methods}

Chemicals and reagents. Bovine serum albumin (BSA), D-glucose and rabbit anti-GAPDH antibody were purchased from Sigma-Aldrich (Merck KGaA, Darmstadt, Germany). Rabbit anti-profilin-1 and anti-Rho antibodies were purchased from Abcam (Shanghai, China), mouse anti-RAGE antibody was purchased from Santa Cruz Biotechnology, Inc. (Dallas, TX, USA) and rabbit anti-p65 antibody was obtained from Bioworld Technology, Inc. (St. Louis Park, MN, USA). Blood glucose and total cholesterol (TC) detection kits were obtained from Nanjing Jiancheng Bioengineering Institute (Nanjing, China). The procollagen type III N-terminal peptide (PIIINP) ELISA kit was purchased from Uscn Life Sciences, Inc. (Wuhan, China). TRIzol reagent was purchased from Invitrogen (Thermo Fisher Scientific, Inc., Waltham, MA, USA). The reverse transcription kit was obtained from Thermo Fisher Scientific, Inc. The QuantiFast SYBR Green PCR kit was from Qiagen GmbH (Hilden, German). The profilin-1 short hairpin RNA (shRNA) adenovirus vectors and blank control adenovirus vectors were designed and synthesized by Hanbio Biotechnology Co., Ltd. (Shanghai, China).

Preparation of AGEs. AGEs were prepared according to the protocol described by Wu et al (22). Briefly, BSA $(10 \mathrm{mg} / \mathrm{ml})$ was incubated in 0.2 M PBS with D-glucose (90 g/l) containing $100 \mathrm{U} / \mathrm{ml}$ penicillin and $100 \mu \mathrm{g} / \mathrm{ml}$ streptomycin in the dark at $37^{\circ} \mathrm{C}$ for 12 weeks. After 12 weeks incubation, the preparations were dialyzed three times for $18 \mathrm{~h}$ at $4^{\circ} \mathrm{C}$ against PBS ( $\mathrm{pH}$ 7.4) each time to remove free glucose, and were subsequently separated into aliquots and stored at $-20^{\circ} \mathrm{C}$ prior to use.

Animal protocol. Male ( $\mathrm{n}=30$; weight, $150 \pm 10 \mathrm{~g}$; age, 1 month) Sprague-Dawley rats were purchased from Shanghai SLAC Laboratory Animal Co., Ltd. (Shanghai, China). The rats were raised at an ambient temperature of $24^{\circ} \mathrm{C}$ with 12-h light/dark cycle and $50 \pm 5 \%$ humidity, and free access to a standard chow diet and water. Rats were randomly divided into the following four groups ( $n=6$ each): Control, rats were treated with vehicle saline; AGEs, rats were treated with AGEs; AGEs + S, rats were treated with AGEs and profilin-1 shRNA adenovirus vectors; and AGEs $+\mathrm{V}$, rats were treated with AGEs and blank control adenovirus vectors. For transfection efficiency detection, the remaining 6 rats were randomly divided into two groups $(n=3$ each) for 4 weeks treatment: Ad-vector group, rats were treated with blank control adenovirus vector; Ad-profilin-1 shRNA group, rats were treated with adenovirus vector containing profilin-1 shRNA. AGEs ( $50 \mathrm{mg} / \mathrm{kg} /$ day) was administered by tail vein injection for 8 weeks, with the same volume of saline injected as a control. Ad-profilin-1 shRNA or blank control vector was injected twice by tail vein at a dose of $3 \times 10^{9}$ plaque forming units with an interval of every 4 weeks, beginning with the initial AGEs injection. The experimental procedures and protocols performed in the present study were approved by the Medicine Animal Welfare Committee of Xiangya Hospital, Central South University (Changsha, China), conforming with the National Institutes of Health Guide for the Care and Use of Laboratory Animals (NIH publication 85-23, revised 1996) (23).

Echocardiography. The cardiac contractile function in vivo was determined after 8 weeks of daily administration of AGEs (50 mg/kg/day) using a BL-420E Data Acquisition and Analysis system (Chengdu TME Technology Co., Ltd., Chengdu, China). Following anesthesia with pentobarbital sodium (60 mg/kg; intraperitoneal), all rats were examined and, using the left ventricular long axis view, the following parameters were measured: Left ventricular ejection fraction (LVEF) and left ventricular fractional shortening (LVFS).

Preparation of serum and tissue samples. After 8 weeks of daily administration of AGEs (50 mg/kg/day), $800 \mu \mathrm{l}$ blood 
Table I. Sequences of reverse transcription-quantitative polymerase chain reaction primers.

\begin{tabular}{lll} 
Gene name & \multicolumn{1}{c}{ Forward primer (5'-3') } & \multicolumn{1}{c}{ Reverse primer (5'-3') } \\
\hline Atrial natriuretic peptide & CTCCGATAGATCTGCCCTCTTGAA & GGTACCGGAAGCTGTTGCAGCCTA \\
MMP-2 & GAAAGGTGCTGACCGTATCC & CCAGTGCCCTCCTAAGACAG \\
MMP-9 & GCCGACTTATGTGGTCTTCC & TGCCCGAGTGTAACCATAGC \\
Profilin-1 & CTGATGGGCAAAGAAGGTC & GGGAAGGGACAGATGAGGTC \\
$\beta$-myosin heavy chain & CCAGAAGCCTCGAAATGTC & CTTTCTTTGCCTTGCCTTTGC \\
GAPDH & CCATGTTCGTCATGGGTGTGAACCA & GCCAGTAGAGGCAGGGATGATGTTC
\end{tabular}

MMP, matrix metalloproteinase.

samples were collected and centrifuged for $10 \mathrm{~min}$ at $4^{\circ} \mathrm{C}$ and $3,000 \mathrm{x}$ g. Supernatants were stored at $-80^{\circ} \mathrm{C}$ until the analysis of biochemistry parameters. The whole heart was harvested immediately following blood sample selection, the atrium and right ventricle were removed, and left ventricle samples were divided into the following three groups: One group was fixed with $4 \%$ paraformaldehyde at room temperature for 1 week and embedded in paraffin. Paraffin sections were used for Masson's trichrome staining and immunohistochemistry; the second group was homogenized by TRIzol reagent for reverse transcription-quantitative polymerase chain reaction (RT-qPCR) analysis; the final group was homogenized in radioimmunoprecipitation assay lysis buffer (P0013B; Beyotime Institute of Biotechnology, Beijing, China) and $0.1 \mathrm{mmol} / \mathrm{l}$ phenylmethylsulfonyl fluoride (ST506; Beyotime Institute of Biotechnology) for western blot analysis.

Measurement of metabolic parameters and PIIINP. Metabolic parameters, including blood glucose and TC, were detected using relevant kits. The serum levels of PIIINP were detected using an ELISA kit (cat. no. SEA573Ra), according to the manufacturer's instructions.

Protein preparation and western blotting. Total protein from left ventricle tissue samples was extracted, as described above for the preparation of serum and tissue samples, and measured using a BCA protein assay kit. Following heating at $95^{\circ} \mathrm{C}$ for $5 \mathrm{~min}, 20 \mu \mathrm{g} / \mathrm{sample}$ lysates were separated by 10 or $12 \%$ SDS-PAGE and transferred to polyvinylidene fluoride membranes. The membranes were blocked with $5 \%$ fat-free milk in TBST buffer $(0.1 \%$ Tween-20 in TBS) for 90 min at room temperature, and subsequently incubated with primary antibodies against profilin-1 (cat. no. ab124904, 1:1,500 dilution), p65 (cat. no. BS1253, 1:1,000 dilution), RAGE (cat. no. sc-365154, 1:600 dilution), Rho (cat. no. ab40673, 1:1,000 dilution) and GAPDH (cat. no. G9545, 1:5,000 dilution) at $4{ }^{\circ} \mathrm{C}$ overnight. Following primary antibody incubation, membranes were subsequently washed and incubated with horseradish peroxidase-conjugated goat anti-mouse (cat. no. CW0102, 1:5,000 dilution; CW Biotech, Co., Ltd., Beijing, China) or anti-rabbit IgG (cat. no. CW0103, 1:5,000 dilution; $\mathrm{CW}$ Biotech) secondary antibodies for $1 \mathrm{~h}$ at room temperature. Potent ECL kit (cat. no. 70-P1425; MultiSciences Biotech Co., Ltd., Hangzhou, China) was used to visualize proteins. The signal was detected and ratios of the target protein against the GAPDH control were calculated using the Image Lab software (version 5.2.1; Bio-Rad Laboratories, Inc., Hercules, CA, USA).

$R N A$ isolation and RT-qPCR. Total RNA was extracted from left ventricle tissue samples using TRIzol reagent, according to the manufacturer's instructions. The concentration and purity of isolated RNA was subsequently detected. cDNA was generated from $1 \mu \mathrm{g}$ total RNA using a high-capacity cDNA reverse transcription kit (4368814; Thermo Fisher Scientific, Inc.) following the manufacturer's instructions. Reverse transcription was performed as follows: $25^{\circ} \mathrm{C}$ for $10 \mathrm{~min}, 37^{\circ} \mathrm{C}$ for $2 \mathrm{~h}, 85^{\circ} \mathrm{C}$ for $5 \mathrm{~min}$ and stored at $4^{\circ} \mathrm{C}$. GAPDH primers were purchased from Sangon Biotech Co., Ltd. (Shanghai, China) and all other primers were synthesized and purified by Sangon Biotech Co., Ltd., as listed in Table I. cDNA was quantified using a QuantiFast SYBR Green PCR kit and qPCR was performed using a 7500 Fast Real-Time PCR System (Applied Biosystems; Thermo Fisher Scientific, Inc.). Briefly, the amplification was performed with an initial step at $95^{\circ} \mathrm{C}$ for $5 \mathrm{~min}$, followed by 40 cycles of denaturation at $95^{\circ} \mathrm{C}$ for $10 \mathrm{sec}$, annealing at $60^{\circ} \mathrm{C}$ for $30 \mathrm{sec}$ and extension at $60^{\circ} \mathrm{C}$ for $30 \mathrm{sec}$ for each target gene. All amplification reactions for each sample were performed in triplicate and the results were expressed as the ratio of target genes to GAPDH mRNA using the $2^{-\Delta \Delta C q}$ method (24).

Histology and Immunohistochemistry analysis. Paraformaldehyde-fixed paraffin sections were cut from the left ventricle at a thickness of $4 \mu \mathrm{m}$ and were stained using a Masson's trichrome stain kit (BSBA-4079A; Zhongshan Golden Bridge Biotechnology Co., Beijing, China), according to the manufacturer's instructions, to evaluate ventricular remodeling. Immunohistochemistry was performed as described previously (14). Briefly, left ventricle sections, at a thickness of $4 \mu \mathrm{m}$, were incubated overnight at $4^{\circ} \mathrm{C}$ with the primary antibody against profilin-1 (1:200 dilution), and subsequently incubated with a biotin conjugated goat anti-rabbit immunoglobulin-G secondary antibody (cat. no. ZB-2010; Zhongshan Golden Bridge Biotechnology Co., Beijing, China) at a $1: 1,000$ dilution at $37^{\circ} \mathrm{C}$ for $20 \mathrm{~min}$. Following washing in PBS, sections were developed using 3,3'-diaminobenzidine tetrahydrochloride obtained from Zhongsha Golden Bridge Biotechnology Co. (cat. no. ZLI-9018) and counterstained with 50\% hematoxylin for 
Table II. Biochemical characteristics of rats following various treatments.

\begin{tabular}{|c|c|c|c|c|}
\hline & Control & AGEs & AGEs $+\mathrm{S}$ & AGEs + V \\
\hline Body weight, g & $552.2 \pm 1.7$ & $554.5 \pm 4.6$ & $554.3 \pm 3.3$ & $553.3 \pm 2.7$ \\
\hline Glucose, $\mathrm{mmol} / \mathrm{l}$ & $3.9 \pm 0.6$ & $4.4 \pm 0.5$ & $4.0 \pm 0.6$ & $4.1 \pm 0.6$ \\
\hline Total cholesterol, mmol/1 & $3.1 \pm 0.2$ & $3.6 \pm 0.8$ & $3.2 \pm 0.5$ & $3.3 \pm 0.5$ \\
\hline
\end{tabular}

Data are presented as the mean \pm standard deviation, $\mathrm{n}=6$. AGEs, advanced glycation end products; shRNA, short hairpin RNA; control, rats treated with vehicle saline; AGEs, rats treated with AGEs; AGEs + S, rats treated with AGEs + profilin-1 shRNA adenovirus; AGEs + V, rats treated with AGEs + control adenovirus vectors.

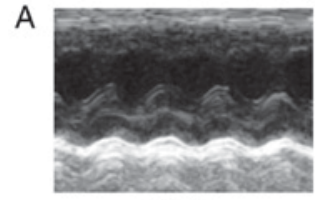

Control

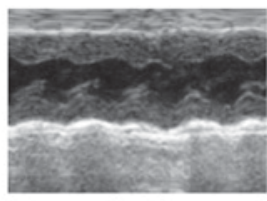

AGES+S

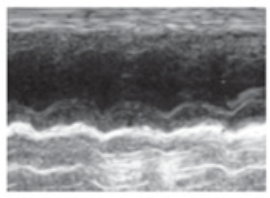

AGEs

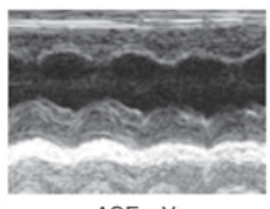

AGES+V
B

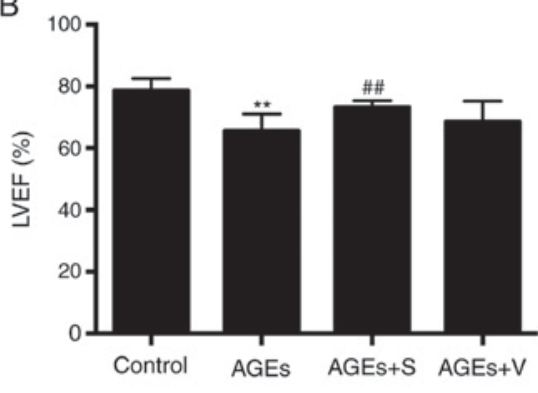

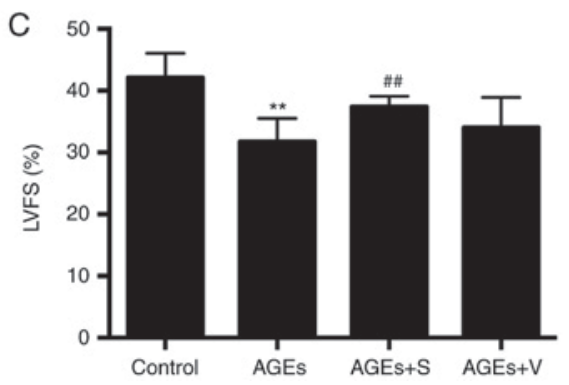

Figure 1. The effect of profilin-1 shRNA adenovirus on cardiac function induced by chronic delivery of AGEs. (A) Representative echocardiography images demonstrate the left cardiac function after 8 weeks. (B) LVEF after 8 weeks of chronic delivery of AGEs. (C) LVFS after 8 weeks of chronic delivery of AGEs. Data are presented as the mean + standard deviation, $n=6$ per group. ${ }^{* *} \mathrm{P}<0.01$ vs. control; ${ }^{\# \#} \mathrm{P}<0.05$ vs. AGEs-only group. shRNA, short hairpin RNA; AGEs, advanced glycation end products; LVEF, left ventricular ejection fraction; LVFS, left ventricular fractional shortening; control, rats treated with vehicle saline; AGEs, rats treated with AGEs; AGEs + S, rats treated with AGEs + profilin-1 shRNA adenovirus; AGEs + V, rats treated with AGEs + blank control adenovirus vectors.

$15 \mathrm{~min}$ at room temperature. Subsequently, sections were mounted with neutral balsam. Masson's trichrome stain and immunohistochemistry pictures were visualized under a light microscope (magnification, x200; Nikon Corporation, Tokyo, Japan).

Statistical analysis. Data are presented as the mean \pm standard deviation. Statistical analysis was performed using SPSS 17.0 software (SPSS, Inc., Chicago, IL, USA) either by unpaired student's t-test for two groups or one-way analysis of variance followed by Newman-Keuls post-hoc test for multiple groups. $\mathrm{P}<0.05$ was considered to indicate a statistically significant difference.

\section{Results}

Characteristics of the exogenous AGE infusion rat model. As presented in Table II, no significant differences were observed in the body weight, blood glucose and TC of different groups of rats following 8 weeks of treatment.

Chronic injection of AGEs causes cardiac contractile dysfunction, hypertrophy and fibrosis, and increases the expression of profilin-1. To determine whether chronic injection of AGEs has an effect on cardiac injury, the present study focused on the primary characteristics of DCM, including cardiac function, fibrosis and hypertrophy. Compared with the control group, echocardiography demonstrated that left ventricle systolic function, including LVEF and LVFS, was significantly decreased in the AGEs group (Fig. 1). In addition, the AGEs group developed cardiac fibrosis, as evidenced by markedly increased collagen deposition in the heart tissue sections, particularly around vessels, indicated by blue color in Masson's trichrome staining (Fig. 2A). Furthermore, PIIINP expression in the serum, and MMP-2 and -9 mRNA expression in left ventricle tissues, was significantly increased in the AGEs 
A

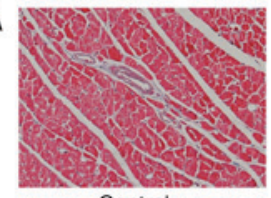

Control

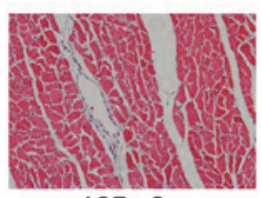

AGES+S

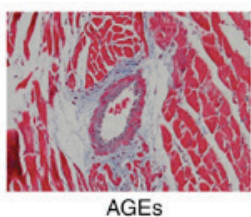

AGEs

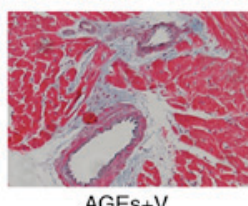

AGEs+V
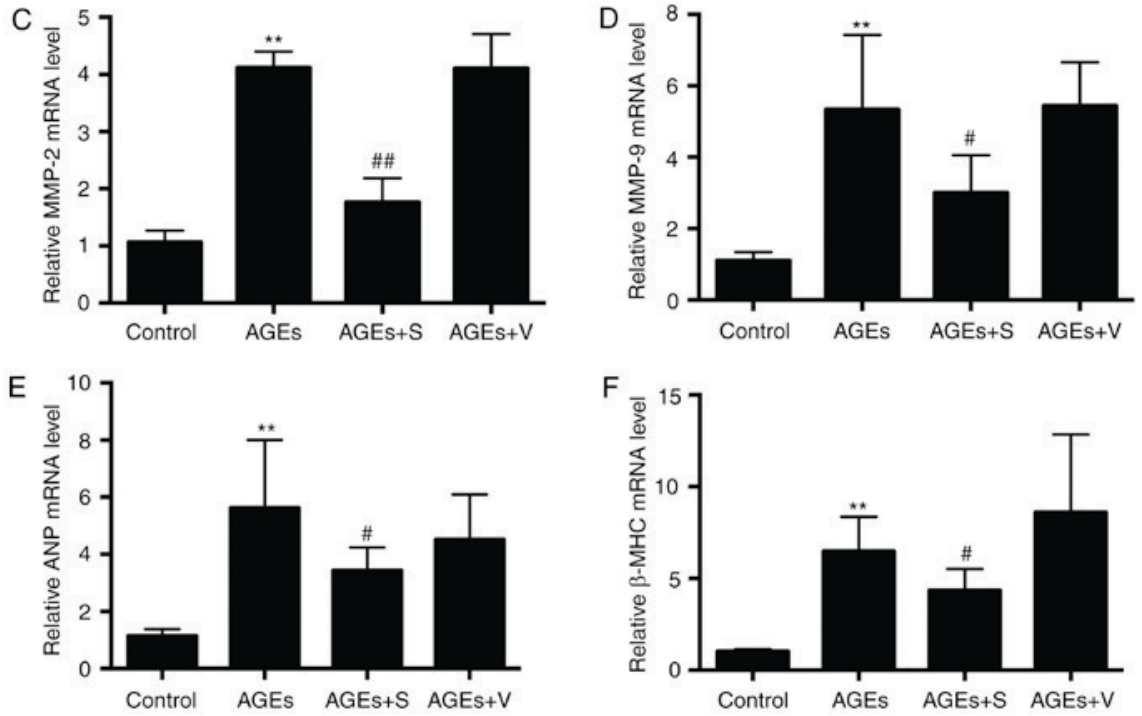

Figure 2. The effect of profilin-1 shRNA adenovirus on cardiac remodeling induced by chronic delivery of AGEs. (A) Representative Masson's trichrome staining images indicate the fibrotic areas in left ventricles of hearts. Magnification, x200. (B) Expression of PIIINP in the serum. mRNA expression of (C) MMP-2, (D) MMP-9, (E) ANP and (F) $\beta$-MHC in left ventricle heart tissues. Data are presented as the mean \pm standard deviation, $\mathrm{n}=6 / \mathrm{group}$. $\mathrm{P}<0.05$ and ${ }^{* *} \mathrm{P}<0.01$ vs. control; ${ }^{\#} \mathrm{P}<0.05$ and ${ }^{\# \#} \mathrm{P}<0.01$ vs. AGEs-only group. shRNA, short hairpin RNA; AGEs, advanced glycation end products; PIIINP, procollagen type III N-terminal peptide; MMP, matrix metalloproteinase; ANP, atrial natriuretic peptide; $\beta$-MHC, $\beta$-myosin heavy chain; control, rats treated with vehicle saline; AGEs, rats treated with AGEs; AGEs + S, rats treated with AGEs + profilin-1 shRNA adenovirus; AGEs + V, rats treated with AGEs + blank control adenovirus vectors.

group compared with the control (Fig. 2B-D). As demonstrated in Fig. 2E and F, AGEs significantly increased the expression of atrial natriuretic peptide (ANP) and $\beta$-myosin heavy chain $(\beta-\mathrm{MHC})$ compared with the control, which indicates cardiac hypertrophy. As expected, chronic delivery of AGEs significantly increased the expression of profilin-1 compared with the control group, as determined by immunohistochemistry, western blot analysis and RT-qPCR (Fig. 3). No marked differences were observed between AGEs and AGEs + V groups (Figs. 1-3).

Downregulation of profilin-1 expression attenuates AGE-induced cardiac dysfunction, hypertrophy and fibrosis. To investigate the role of profilin-1 in AGE-induced cardiac dysfunction, hypertrophy and fibrosis, the present study knocked down profilin-1 expression by intravenous delivery of adenovirus expressing profilin-1 shRNA. On day 6 after the second injection of adenovirus, 50 and $40 \%$ decreases in profilin-1 expression at protein and mRNA levels were observed, respectively (Fig. 4A and B). Knockdown of profilin-1 expression improved cardiac systolic function (Fig. 1) and reduced cardiac fibrosis, as indicated by reduced collagen deposition (Fig. 2A) and reduced PIIINP, MMP-2 and MMP-9 expression (Fig. 2B-D), compared with the AGEs-only group. In addition, silencing profilin-1 expression significantly inhibited AGE-induced cardiac hypertrophy, as indicated by reduced ANP and $\beta$-MHC expression (Fig. $2 \mathrm{E}$ and F) compared with the AGEs-only group.

Nuclear factor (NF)- $\kappa B$ and Rho signaling pathways are involved in AGE-induced cardiac injury. Increasing evidence has indicated that AGEs activate various signaling pathways, including $\mathrm{NF}-\kappa \mathrm{B}$ and Rho/Rho-associated protein kinase (ROCK), and induces the expression of genes associated with diabetic complications by binding to RAGE $(2,25,26)$. Due to the important role of the NF- $\mathrm{B}$ signaling pathway in regulating oxidative stress, fibrosis, hypertrophy and apoptosis, and as the Rho/ROCK pathway in the actin cytoskeleton are reported to be involved DCM, p65 and Rho were selected for further investigation in the present study. Following chronic treatment with AGEs for 8 weeks, a significant increase in the expression of RAGE, p65 and Rho at the protein level was observed in left ventricle heart tissues, compared with the control group (Fig. 5). Notably, blockade of profilin-1 significantly reduced the expression of RAGE, p65 and Rho compared with the AGEs-only group (Fig. 5). 
A

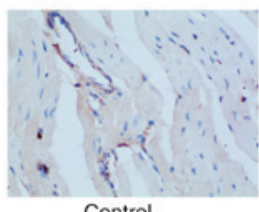

Control

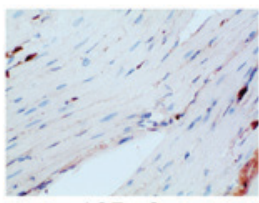

AGEs+S

C

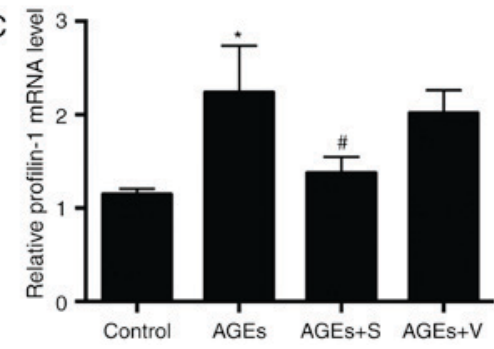

B

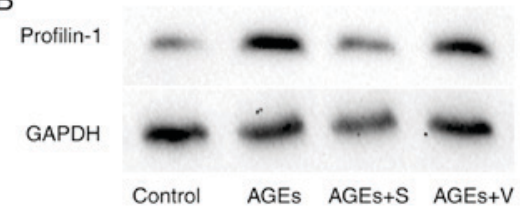

Control AGEs AGEs+S AGEs+V

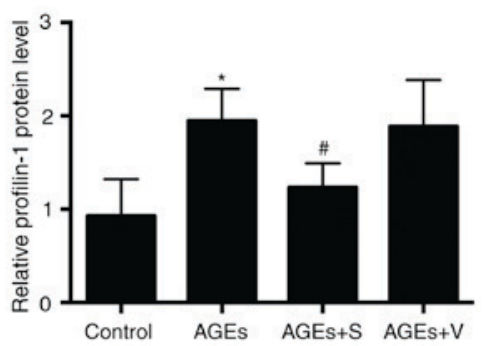

Figure 3. Effect of adenovirus on the expression of profilin-1 in AGE-treated heart tissues. (A) Representative immunohistochemical staining images demonstrate profilin-1 expression in AGE-treated and control left ventricle heart tissues. Magnification, x200. (B) Profilin-1 protein expression in AGE-treated and control left ventricle heart tissues, as measured by western blot analysis. (C) mRNA expression of profilin-1 in AGE-treated and control left ventricle heart tissues. Data are presented as the mean \pm standard deviation, $\mathrm{n}=6 /$ group. ${ }^{*} \mathrm{P}<0.05$ vs. control; ${ }^{\text {P }}<0.05$ vs. AGEs-only group. AGEs, advanced glycation end products; control, rats treated with vehicle saline; AGEs, rats treated with AGEs; AGEs + S, rats treated with AGEs + profilin-1 shRNA adenovirus; AGEs + V, rats treated with AGEs + blank control adenovirus vectors.

A

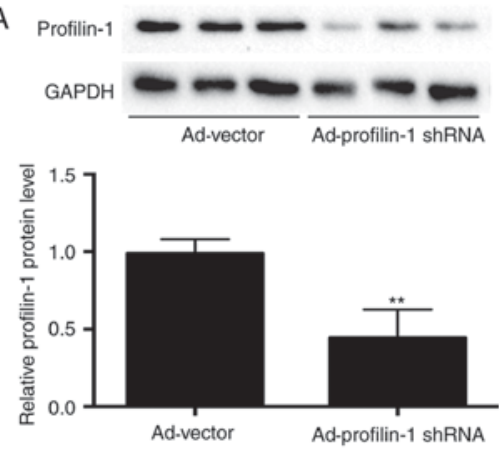

$\mathrm{B}$

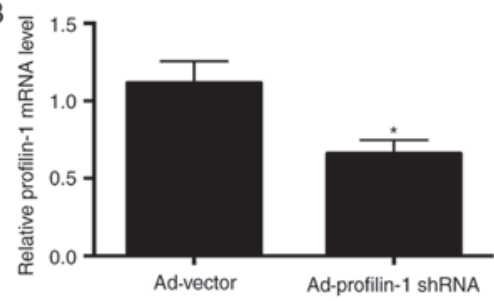

Figure 4. Effect of adenovirus on the expression of profilin-1 in normal heart tissues. (A) Effect of adenovirus on the expression of profilin-1 in normal heart tissue at the protein level, as measured by western blot analysis. (B) Effect of adenovirus on the expression of profilin-1 in normal heart tissue at the mRNA level. Data are presented as the mean + standard deviation, $\mathrm{n}=3$ /group, ${ }^{*} \mathrm{P}<0.05$ and ${ }^{* *} \mathrm{P}<0.01$ vs. Ad-vector group. Ad, adenovirus; shRNA, short hairpin RNA; Ad-vector, rats treated with blank control adenovirus vectors; Ad-profilin-1 shRNA, rats treated with profilin-1 shRNA adenovirus.

\section{Discussion}

The primary findings of the present study were as follows: Chronic injection of AGEs markedly upregulated the expression of profilin-1 in vivo, which was associated with abnormal cardiac structure and function, potentially via the activation of NF- $\kappa \mathrm{B}$ and Rho signaling pathways; and knocking down the expression of profilin-1 attenuated AGE-induced myocardial injury, including cardiac dysfunction, hypertrophy and fibrosis. These results indicate that profilin-1 may represent a crucial mediator in AGE-induced cardiac injury, which may be developed as a potential therapeutic target for patients with diabetes to protect against heart failure.

Cardiac hypertrophy and fibrosis are the major features of DCM, and result in ventricular dysfunction and heart failure $(27,28)$. Evidence has indicated that increased and accelerated formation of AGEs has emerged as an important contributor to the onset and development of cardiac hypertrophy and fibrosis (2). Thus, identifying the mechanisms mediated by AGEs is essential for the development of novel therapeutic targets for the treatment of DCM. It was previously reported that intraperitoneal or tail vein injection of AGEs caused vascular impairment and remodeling $(29,30)$, and $\sim 70 \%$ of the injected ${ }^{125} \mathrm{I}-\mathrm{AGE}$ irreversibly bound to heart muscle tissues (30). Therefore, we hypothesized that increasing the circulating concentration of AGEs by tail vein injection may mimic myocardial injury in vivo. The results of the present study demonstrated that chronic injection of exogenous AGEs for 8 weeks markedly decreased cardiac contractile function, and induced cardiac hypotrophy and fibrosis (Figs. 1 and 2). However, no alterations in the circulating levels of glucose and lipids were observed (Table II), which indicates that AGEs 

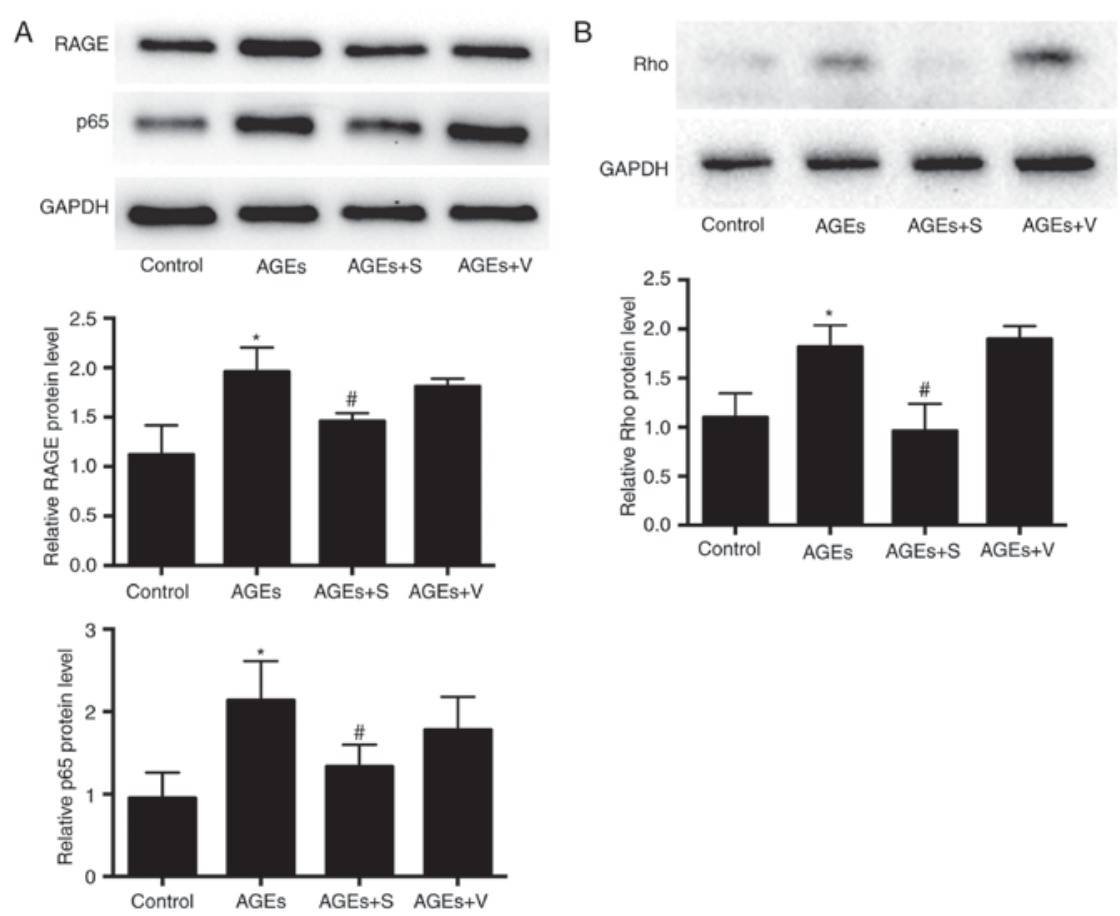

Figure 5. Nuclear factor- $\kappa \mathrm{B}$ and Rho signaling pathways are involved in cardiac injury induced by AGEs. (A) Representative western blot and quantified protein expression for RAGE and p65. (B) Representative western blot and quantified protein expression for Rho. Data are presented as the mean \pm standard deviation, $\mathrm{n}=6$ /group. ${ }^{*} \mathrm{P}<0.05$ vs. control; ${ }^{\text {}} \mathrm{P}<0.05$ vs. AGEs-only group. AGEs, advanced glycation end products; RAGE, receptor for AGEs; control, rats treated with vehicle saline; AGEs, rats treated with AGEs; AGEs + S, rats treated with AGEs + profilin-1 shRNA adenovirus; AGEs + V, rats treated with AGEs + blank control adenovirus vectors.

may have an independent effect on myocardium injury, in the absence of hyperglycemia and hyperlipidemia.

It is established that profilin-1 is a highly conserved actin binding protein that has a prominent role in various cellular processes (10). Increasing evidence indicates that, in addition to regulating actin polymerization, profilin-1 is an important molecule associated with pathological hypertrophy and fibrosis. It was previously reported that profilin-1 was upregulated 2-3-fold in hypertrophic vascular tissues and ventricular cardiomyocytes $(17,18)$, and overexpression of profilin-1 directly caused vascular and cardiac hypertrophy and fibrosis by activating mitogen-activated protein kinase or extracellular signal-regulated kinase $1 / 2$ signaling pathways (17). By contrast, suppression of profilin-1 expression conferred protection against hypertrophic insult. Notably, profilin-1 overexpression-associated ventricular hypertrophy is not only observed in the ultra-early stage (21), but also at later stages (19), which indicates that profilin-1 may function as an important regulator throughout the progression of the pathological cardiac hypertrophy. In the present study, the results demonstrated that chronic injection of AGEs significantly upregulated the mRNA and protein expression of profilin-1 in the heart, which was accompanied by cardiac hypertrophy, fibrosis and impaired heart function. In addition, AGE-induced cardiac fibrosis was particularly prominent in the area surrounding vessels, which is a feature of both human patients and animal models of DCM (27). However, silencing profilin-1 expression markedly attenuated the observed AGE-induced cardiac injury.

Previous studies have provided evidence that demonstrates that the activation of $\mathrm{NF}-\kappa \mathrm{B}$ and Rho/ROCK signaling pathways in the heart contributes to the development of DCM, and targeting $\mathrm{NF}-\kappa \mathrm{B}$ and Rho/ROCK signaling may improve diabetic cardiac function in patients with diabetes (31-35). The results of the present study demonstrated that chronic intravenous delivery of exogenous AGEs increased the protein expression of p65 and Rho in heart tissue, and increased protein expression of RAGE was also observed, compared with the control group. Our previous study indicated that profilin-1 may function as a common cellular molecule, and may be downstream of protein kinase $\mathrm{C}$ or $\mathrm{NF}-\kappa \mathrm{B}$ signaling pathways mediated by AGEs in endothelial cells (12). Therefore, we hypothesized that AGEs may activate NF- $\kappa \mathrm{B}$ and Rho signaling pathways via RAGE, which results in increased profilin-1 expression. Notably, in the present study, inhibition of profilin-1 expression decreased the protein expression of p65 and Rho, and ameliorated cardiac injury, which indicates a potential positive feedback loop between profilin-1 and p65, and profilin-1 and Rho. Previous studies have demonstrated that in diabetic cardiomyocytes, actin polymerization is significantly increased (32), and the actin cytoskeleton has an important role in sustaining ROS production (31). Therefore, it may be inferred that increased profilin-1 expression induced by AGEs may cause accumulated actin polymerization and, in turn, excess ROS production, which subsequently activates $\mathrm{NF}-\kappa \mathrm{B}$ and Rho to form a positive loop.

In conclusion, the present study demonstrates that chronic delivery of AGEs promoted cardiac fibrosis and hypertrophy, impaired systolic function and upregulated profilin-1 expression, which may occur via NF- $\mathrm{BB}$ or Rho signaling pathways. Reducing profilin-1 expression may aid the prevention of heart injuries in patients with diabetes. Therefore, targeting 
AGE-induced profilin-1 expression may represent a novel therapeutic target to treat diabetic heart failure.

\section{Acknowledgements}

This study was supported by the Central South University Innovation Foundation for postgraduates (grant no. 2015zzts111, awarded to Dr Dafeng Yang), the National Natural Science Foundation of China (grant no. 81000140, awarded to Dr Meifang Chen; grant nos. 81570320 and 81570334, awarded to Dr Tianlun Yang) and the Xiangya Eminent Doctor Project (grant no. 013, awarded to Dr Tianlun Yang). The authors thank Dr Xinghui Sun (Department of Biochemistry, University of Nebraska, Lincoln, NE, USA) and Dr Shao Liu (Department of Pharmacy, Xiangya Hospital, Central South University, Changsha, China) for their assistance with revision of the manuscript.

\section{References}

1. Miki T, Yuda S, Kouzu H and Miura T: Diabetic cardiomyopathy: Pathophysiology and clinical features. Heart Fail Rev 18: 149-166, 2013

2. Bodiga VL, Eda SR and Bodiga S: Advanced glycation end products: Role in pathology of diabetic cardiomyopathy. Heart Fail Rev 19: 49-63, 2014

3. Sveen KA, Nerdrum T, Hanssen KF, Brekke M, Torjesen PA, Strauch CM, Sell DR, Monnier VM, Dahl-Jørgensen K and Steine K: Impaired left ventricular function and myocardia blood flow reserve in patients with long-term type 1 diabetes and no significant coronary artery disease: Associations with protein glycation. Diab Vasc Dis Res 11: 84-91, 2014.

4. Cooper ME: Importance of advanced glycation end products in diabetes-associated cardiovascular and renal disease. Am J Hypertens 17: 31S-38S, 2004.

5. Ko SY, Lin IH, Shieh TM, Ko HA, Chen HI, Chi TC, Chang SS and Hsu YC: Cell hypertrophy and MEK/ERK phosphorylation are regulated by glyceraldehyde-derived AGEs in cardiomyocyte H9c2 cells. Cell Biochem Biophys 66: 537-544, 2013.

6. Li SY, Sigmon VK, Babcock SA and Ren J: Advanced glycation endproduct induces ROS accumulation, apoptosis, MAP kinase activation and nuclear O-GlcNAcylation in human cardiac myocytes. Life Sci 80: 1051-1056, 2007.

7. Guo R, Liu W, Liu B, Zhang B, Li W and Xu Y: SIRT1 suppresses cardiomyocyte apoptosis in diabetic cardiomyopathy: An insight into endoplasmic reticulum stress response mechanism. Int J Cardiol 191: 36-45, 2015.

8. Brouwers O, de Vos-Houben JM, Niessen PM, Miyata T, van Nieuwenhoven F, Janssen BJ, Hageman G, Stehouwer CD and Schalkwijk CG: Mild oxidative damage in the diabetic rat heart is attenuated by glyoxalase-1 overexpression. Int J Mol Sci 14: 15724-15739, 2013.

9. Pernier J, Shekhar S, Jegou A, Guichard B and Carlier MF: Profilin interaction with actin filament barbed end controls dynamic instability, capping, branching, and motility. Dev Cell 36: 201-214, 2016.

10. Witke W: The role of profilin complexes in cell motility and other cellular processes. Trends Cell Biol 14: 461-469, 2004.

11. Jockusch BM, Murk K and Rothkegel M: The profile of profilins. Rev Physiol Biochem Pharmacol 159: 131-149, 2007.

12. Li Z,Zhong Q, Yang T, Xie X and Chen M: The role of profilin- 1 in endothelial cell injury induced by advanced glycation end products (AGEs). Cardiovasc Diabetol 12: 141, 2013.

13. Romeo G, Frangioni JV and Kazlauskas A: Profilin acts downstream of LDL to mediate diabetic endothelial cell dysfunction. FASEB J 18: 725-727, 2004.

14. Cheng JF, Ni GH, Chen MF, Li YJ, Wang YJ, Wang CL, Yuan Q, Shi RZ, Hu CP and Yang TL: Involvement of profilin-1 in angiotensin II-induced vascular smooth muscle cell proliferation. Vascul Pharmacol 55: 34-41, 2011.

15. Jin HY, Song B, Oudit GY, Davidge ST, Yu HM, Jiang YY, Gao PJ, Zhu DL, Ning G, Kassiri Z, et al: ACE2 deficiency enhances angiotensin II-mediated aortic profilin-1 expression, inflammation and peroxynitrite production. PLoS One 7: e38502, 2012.
16. Caglayan E, Romeo GR, Kappert K, Odenthal M, Südkamp M, Body SC, Shernan SK, Hackbusch D, Vantler M, Kazlauskas A and Rosenkranz S: Profilin-1 is expressed in human atherosclerotic plaques and induces atherogenic effects on vascular smooth muscle cells. PLoS One 5: e13608, 2010.

17. Kooij V, Viswanathan MC, Lee DI, Rainer PP, Schmidt W, Kronert WA, Harding SE, Kass DA, Bernstein SI, Van Eyk JE and Cammarato A: Profilin modulates sarcomeric organization and mediates cardiomyocyte hypertrophy. Cardiovasc Res 110: 238-248, 2016.

18. Zhao SH, Qiu J, Wang Y, Ji X, Liu XJ, You BA, Sheng YP, Li X and Gao HQ: Profilin-1 promotes the development of hypertension-induced cardiac hypertrophy. J Hypertens 31: 576-586, 2013.

19. Elnakish MT, Hassanain HH and Janssen PM: Vascular remodeling-associated hypertension leads to left ventricular hypertrophy and contractile dysfunction in profilin-1 transgenic mice. J Cardiovasc Pharmacol 60: 544-552, 2012.

20. Hein S, Kostin S, Heling A, Maeno Y and Schaper J: The role of the cytoskeleton in heart failure. Cardiovasc Res 45: 273-278, 2000.

21. Zhao SH, Gao HQ, Ji X, Wang Y, Liu XJ, You BA, Cui XP and Qiu J: Effect of ouabain on myocardial ultrastructure and cytoskeleton during the development of ventricular hypertrophy. Heart Vessels 28: 101-113, 2013.

22. Wu S, Song T, Zhou S, Liu Y, Chen G, Huang N and Liu L: Involvement of $\mathrm{Na}^{+} / \mathrm{H}^{+}$exchanger 1 in advanced glycation end products-induced proliferation of vascular smooth muscle cell. Biochem Biophys Res Commun 375: 384-389, 2008.

23. National Institutes of Health Guide for the Care and Use of Laboratory Animals. National Academies Press 85-23 revised, Washington, DC, 1996. https://grants.nih.gov/grants/olaw/ guide-for-the-care-and-use-of-laboratory-animals.pdf.

24. Livak KJ and Schmittgen TD: Analysis of relative gene expression data using real-time quantitative PCR and the 2(-Delta Delta C(T)) method. Methods 25: 402-408, 2001.

25. Nielsen JM, Kristiansen SB, Nørregaard R, Andersen CL, Denner L, Nielsen TT, Flyvbjerg A and Bøtker HE: Blockage of receptor for advanced glycation end products prevents development of cardiac dysfunction in db/db type 2 diabetic mice. Eur J Heart Fail 11: 638-647, 2009.

26. Ma H, Li SY, Xu P, Babcock SA, Dolence EK, Brownlee M, Li J and Ren J: Advanced glycation endproduct (AGE) accumulation and AGE receptor (RAGE) up-regulation contribute to the onset of diabetic cardiomyopathy. J Cell Mol Med 13: 1751-1764, 2009.

27. Russo I and Frangogiannis NG: Diabetes-associated cardiac fibrosis: Cellular effectors, molecular mechanisms and therapeutic opportunities. J Mol Cell Cardiol 90: 84-93, 2016.

28. Bando YK and Murohara T: Diabetes-related heart failure. Circ J 78: 576-583, 2014.

29. Soro-Paavonen A, Zhang WZ, Venardos K, Coughlan MT, Harris E, Tong DC, Brasacchio D, Paavonen K, Chin-Dusting J, Cooper ME, et al: Advanced glycation end-products induce vascular dysfunction via resistance to nitric oxide and suppression of endothelial nitric oxide synthase. J Hypertens 28: 780-788, 2010.

30. Vlassara H, Fuh H, Makita Z, Krungkrai S, Cerami A and Bucala R: Exogenous advanced glycosylation end products induce complex vascular dysfunction in normal animals: A model for diabetic and aging complications. Proc Natl Acad Sci USA 89: 12043-12047, 1992.

31. Soliman H, Gador A, Lu YH, Lin G, Bankar G and MacLeod KM: Diabetes-induced increased oxidative stress in cardiomyocytes is sustained by a positive feedback loop involving Rho kinase and PKCß2. Am J Physiol Heart Circ Physiol 303: H989-H1000, 2012.

32. Lin G, Craig GP, Zhang L, Yuen VG, Allard M, McNeill JH and MacLeod KM: Acute inhibition of Rho-kinase improves cardiac contractile function in streptozotocin-diabetic rats. Cardiovasc Res 75: 51-58, 2007.

33. Zhou H, Li YJ, Wang M, Zhang LH, Guo BY, Zhao ZS, Meng FL, Deng YG and Wang RY: Involvement of RhoA/ROCK in myocardial fibrosis in a rat model of type 2 diabetes. Acta Pharmacol Sin 32: 999-1008, 2011.

34. Lorenzo O, Picatoste B, Ares-Carrasco S, Ramírez E, Egido J and Tuñón J: Potential role of nuclear factor $\kappa \mathrm{B}$ in diabetic cardiomyopathy. Mediators Inflamm 2011: 652097, 2011.

35. Thomas CM, Yong QC, Rosa RM, Seqqat R, Gopal S, Casarini DE, Jones WK, Gupta S, Baker KM and Kumar R: Cardiac-specific suppression of NF- $\kappa \mathrm{B}$ signaling prevents diabetic cardiomyopathy via inhibition of the renin-angiotensin system. Am J Physiol Heart Circ Physiol 307: H1036-H1045, 2014. 